\title{
Hot filament enhanced chemical vapor deposition of AIN thin films
}

\author{
Jeffrey L. Dupuie and Erdogan Gulari \\ Department of Chemical Engineering, The University of Michigan, Ann Arbor, Michigan 48109
}

(Received 1 February 1991; accepted for publication 6 May 1991)

\begin{abstract}
Hot filament enhanced chemical vapor deposition of aluminum nitride thin films from trimethylaluminum and ammonia has been investigated for deposition temperatures ranging from 584 to $732 \mathrm{~K}$. The use of a hot filament resulted in an approximate two orders of magnitude increase in the deposition rate compared to a similar, uncatalyzed growth. The film deposition rate and refractive index did not depend on the substrate temperature. Xray photoelectron spectroscopy and Fourier-transform infrared spectroscopy indicated that the films were high purity aluminum nitride, with negligible carbon and oxygen contamination.
\end{abstract}

Aluminum nitride (AIN) is a potential dielectric and passivation material for compound semiconductor technology, ${ }^{1-4}$ and also has been investigated for thermal and optical applications due to its large band gap, good thermal stability, and high thermal conductivity. Low temperature growth of aluminum nitride has been achieved by sputtering, plasma-enhanced chemical vapor deposition, and chemical vapor deposition (CVD) from conventional reactants as well as novel precursors. ${ }^{4-7}$ Recent work has shown that AlN films with negligible carbon and oxygen contamination could be deposited from the low temperature, low pressure reaction of trimethylaluminum (TMA) and ammonia. ${ }^{7}$ The films deposited at low temperatures exhibited low refractive indices and low densities compared to films deposited at higher temperatures, however.

Hot filaments have been shown by a number of investigators to be effective in catalyzing the deposition of diamond, ${ }^{8-10}$ and recently have been used to enhance the deposition rate of silicon nitride from monomethylamine. ${ }^{11}$ The purpose of this work was to determine if a hot filament could catalyze the growth of aluminum nitride from trimethylaluminum and ammonia at low temperatures, and to characterize the resultant film properties.

The aluminum nitride films were deposited onto (100) silicon substrates in a cold wall stainless steel chamber. Substrates were pressed by molybdenum clamps against a stainless steel susceptor heated by cartridge heaters. A sheathed type $\mathrm{K}$ thermocouple was used to monitor the susceptor temperature. The susceptor temperature was calibrated under reaction conditions with a type $\mathrm{C}$ thermocouple clamped to the surface of the substrate. $93 \mathrm{~cm}$ of $0.25 \mathrm{~mm}$ O.D. tungsten wire was wrapped around two quartz rods four centimeters from the wafer surface. A conventional organometallic liquid delivery system metered and controlled the trimethylaluminum flow rate. Gases could be introduced into the reactor in either of two delivery lines, one of which could be moved to various points within the chamber, as shown in Fig. 1. The tungsten filament temperature was monitored with a pyrometer. Reported filament temperatures are uncorrected for emissivity and the window material. The deposition parameters used for film growth are given in Table I. Film thicknesses were measured by an ellipsometer at $632.8 \mathrm{~nm}$, and represent an average of 15 points over a 2 in. substrate.

Very little or no growth of aluminum nitride occurred when the tungsten wire was situated between the adjustable organometallic inlet ring and the substrate. Substantial growth was noted on the surface of the inlet ring, however. Rapid growth of film on the substrate was achieved by placing the inlet ring between the hot tungsten filament and the substrate. The deposition rates and refractive indices of the aluminum nitride films as a function of temperature are listed in Table I. The growth rates and refractive indices were insensitive to the wafer temperature. Additional experiments indicated that the film growth rate was proportional to the TMA flow rate, which suggests that the catalyzed deposition process is reactant transport limited.

The decomposition of ammonia on hot platinum filaments has been studied previously. ${ }^{12} \mathrm{NH}$ radicals in the gas phase were detected by laser-induced fluorescence for platinum filament temperatures in excess of $1200 \mathrm{~K}$. The rate of $\mathrm{NH}$ radical production increased with increasing filament temperature, and also with the addition of hydrogen

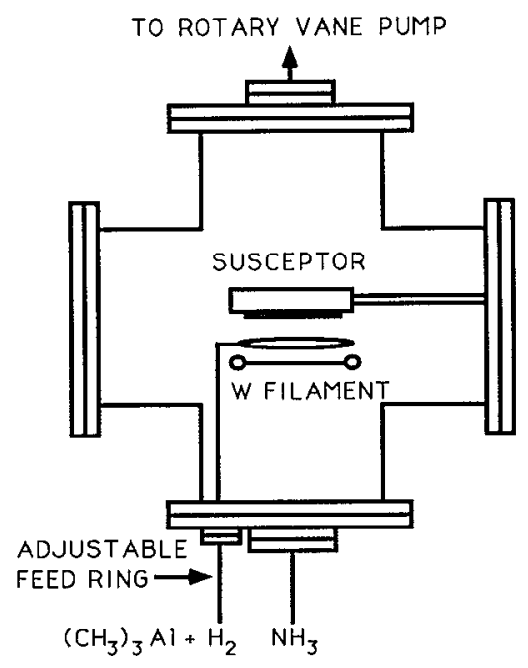

FIG. 1. Schematic of the chemical vapor deposition reactor. 
Sample

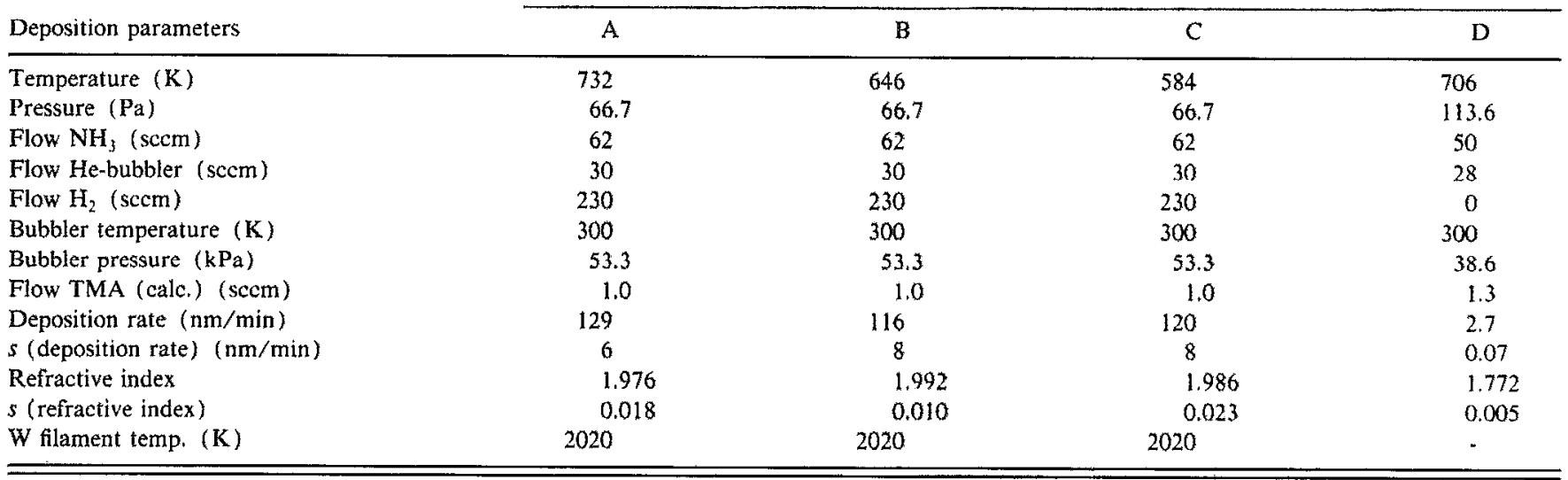

to the gas mixture. In addition to $\mathrm{NH}$, vibrationally excited nitrogen molecules formed by the catalytic decomposition of ammonia on platinum have been detected by molecular beam mass spectrometry. ${ }^{13}$ Similar reactive products formed by the catalytic decomposition of ammonia on tungsten may react with TMA introduced through the gas dispersal ring to form active intermediates, and also may stimulate the desorption of by-products from the growing film surface. The heated tungsten wire enhanced the aluminum nitride growth rate by approximately two orders of magnitude, and resulted in a higher film refractive index compared to a similar uncatalyzed deposition, film $\mathrm{D}$, as
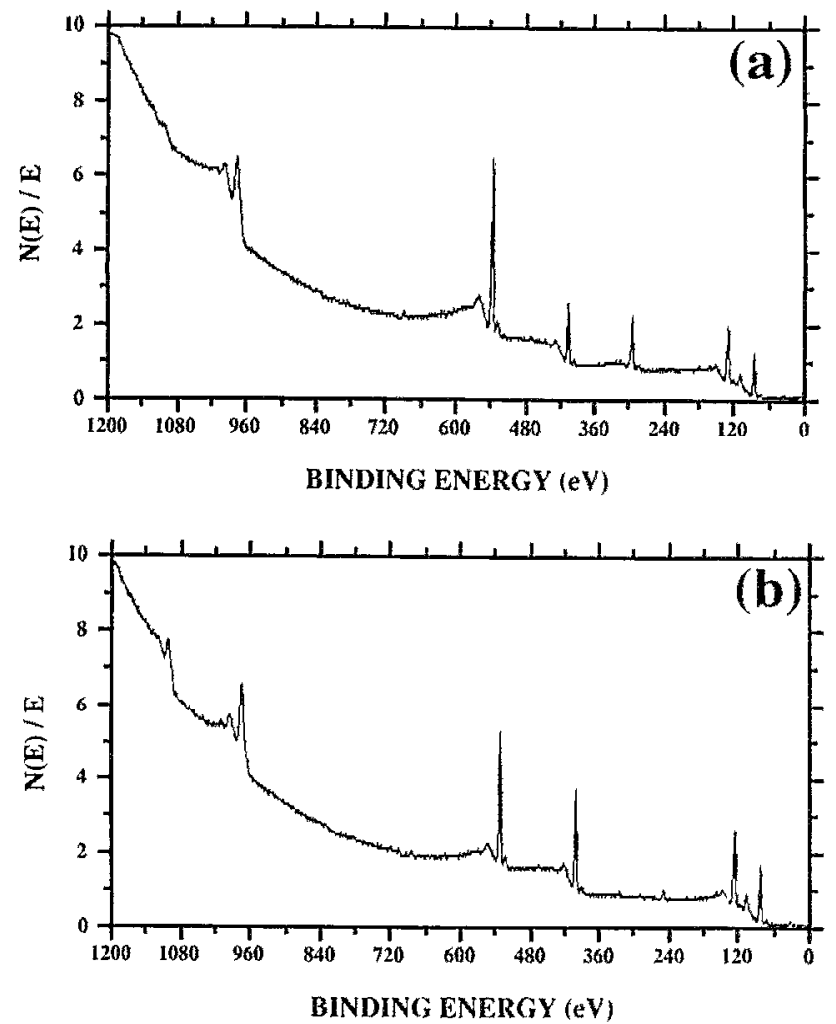

FIG. 2. XPS survey spectra of film C (a) before and (b) after a $30 \mathrm{~s}, 3$ $\mathrm{keV}$ argon ion sputter etch. Note the disappearance of the carbon is peak at $284 \mathrm{eV}$ after the $30 \mathrm{~s}$ sputter etch. indicated in Table I. The deposition temperatures achieved are approximately $300 \mathrm{~K}$ below that reported for the hot filament CVD of silicon nitride. ${ }^{11}$

Figure 2 shows the $\mathrm{x}$-ray photoelectron spectroscopy (XPS) surface spectrum of film $\mathrm{C}$ before and after a $30 \mathrm{~s}$, $3 \mathrm{keV}$ argon ion sputter to remove the surface adventitious carbon. The film deposited at $584 \mathrm{~K}$ with hot filament catalysis contained less than $2 \%$ carbon as determined by XPS. The small carbon signal could have resulted from atomic knock-on caused by the sputtering process. No XPS tungsten signal was observed, the sensitivity for detection being approximately $0.5 \%$. The surface of the film had undergone oxidation, similar to that reported for aluminum nitride deposited by other methods, ${ }^{5.7}$ as well as silicon nitride deposited by thermal CVD. ${ }^{14}$ The oxygen peak underwent rapid atteruation to a negligible level upon further sputtering. The stoichiometry of the sputter profiled film was determined by integration of the XPS photoelectron peak areas with normalization by instrumental atomic sensitivity factors, and was found to be $A 1_{0.52} \mathrm{~N}_{0,48}$.

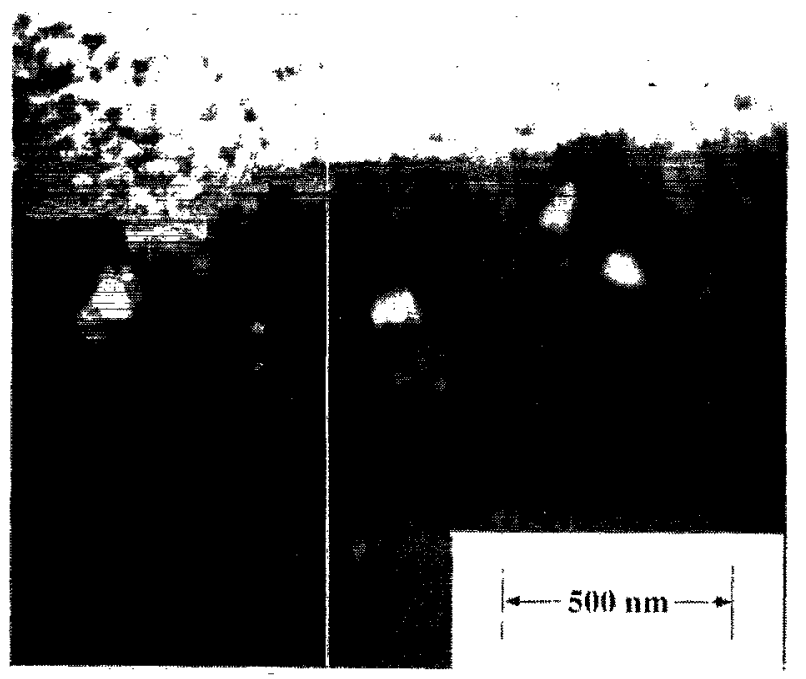

FIG. 3. SEM cross section of film A illustrating the dense columnar growth morphology. 


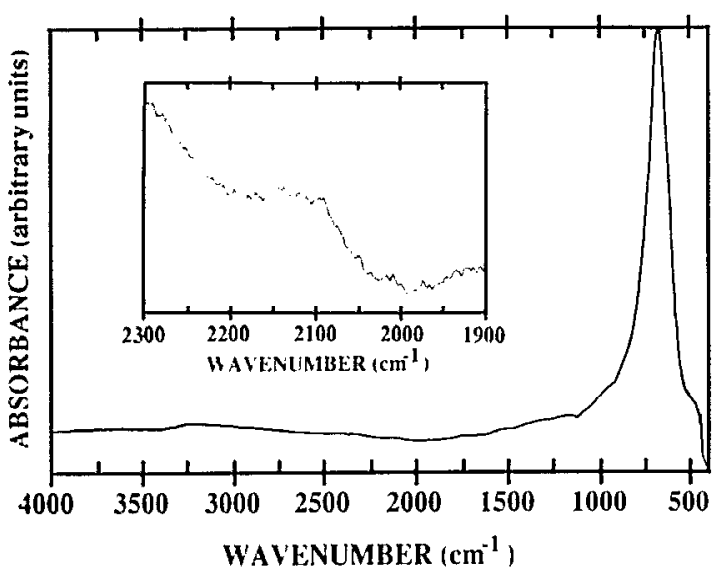

FIG. 4. FTIR spectrum of film A illustrating the strong TO vibration mode of AIN at $663 \mathrm{~cm} .^{-1}$ Inset shows the weak AlN-N absorption at $2100 \mathrm{~cm}$.

The scanning electron microscope (SEM) cross section in Fig. 3 indicates that the aluminum nitride films exhibited a somewhat columnar structure with a slightly rough surface. The surface roughness for a $600 \mathrm{~nm}$ film is on the order of $10 \mathrm{~nm}$.

Figure 4 contains the transmission Fourier-transform infrared (FTIR) spectrum for the AlN film deposited at $732 \mathrm{~K}$. A silicon substrate was used for the background subtraction, and a baseline correction was performed on the spectrum. The strong absorption centered at 663 wavenumbers corresponds to the TO lattice vibration mode of AlN. ${ }^{15}$ A weak absorption band was noted at approximately 2100 wavenumbers, and has been assigned to AIN-N as reported previously. ${ }^{16,17}$ No hydrocarbon absorption bands were noted, indicating that high purity films can be obtained from hot filament enhanced chemical vapor deposition.

In summary, high purity, high refractive index alumi- num nitride thin films were deposited by the tungsten wire catalyzed, low temperature chemical vapor deposition reaction of trimethylaluminum with ammonia. Catalysis of the deposition resulted in deposition rates in excess of 100 $\mathrm{nm} / \mathrm{min}$, an approximate two orders of magnitude enhancement in the deposition rate compared to films deposited under similar conditions without benefit of the hot filament.

The authors would like to thank Beverly Walters for assistance with the SEM work, and Steve Brown for helpful discussions and for the use of the pyrometer. The authors also acknowledge the National Science Foundation, which provided partial financial support for this work.

${ }^{1}$ S. Fujieda, M. Mizuta, and Y. Matsumoto, Jpn. J. Appl. Phys. 27, L296 (1988).

${ }^{2}$ M. Mizuta, S. Fujieda, Y. Matsumoto, and T. Kawamura, Jpn. J. Appl. Phys. 25, L945 (1986).

${ }^{3}$ Y. Mochizuki, M. Mizuta, S. Fujieda, and Y. Matsumoto, Appl. Phys. Lett. 55, 1318 (1989).

${ }^{4}$ F. Hasegawa, T. Takahashi, K. Kubo, and Y. Nannichi, Jpn. J. Appl. Phys. 26, 1555 (1987).

${ }^{5}$ T. K. Hatwar and T. R. Pian, Mater. Res. Soc. Symp. Proc. 121, 557 (1988).

${ }^{\circ}$ W. L. Gladfelter, D. C. Boyd, J. Hwang, R. T. Haasch, J. F. Evans, K. Ho, and K. F. Jensen, Mater. Res. Soc. Symp. Proc. 131, 447 (1989).

${ }^{7}$ J. Dupuie and E. Gulari (unpublished).

${ }^{8}$ Y. Hirose and Y. Terasawa, Jpn. J. Appl. Phys. 25, L519 (1986).

${ }^{9}$ S. Matsumoto, Y. Sato, M. Kamo, and N. Setaka, Jpn. J. Appl. Phys. 21, L183 (1982).

${ }^{10}$ A. Sawabe and T. Inuzuka, Appl. Phys. Lett. 46, 146 (1985).

${ }^{11}$ K. Yasui, H. Katoh, K. Komaki, and S. Kaneda, Appl. Phys. Lett. 56, 898 (1990).

${ }^{12}$ G. S. Selwyn and M. C. Lin, Chem. Phys. 67, 213 (1982).

${ }^{13}$ S. N. Foner and R. L. Hudson, J. Chem. Phys. 80, 518 (1984).

${ }^{14}$ S. I. Raider, R. Flitsch, J. A. Aboaf, and W. A. Pliskin, J. Electrochem. Soc. 123, 560 (1976).

${ }^{15}$ A. T. Collins, E. C. Lightfowlers, and P. J. Dean, Phys. Rev. 158, 833 (1967).

${ }^{10}$ N. Kinoshita, K. Kido, K. Domen, K. Aika, and T. Onishi, J. Chem. Soc. Faraday Trans. 82, 2269 (1986).

${ }^{17}$ M. Oh-kita, K. Aika, K. Urabe, and A. Ozaki, J. Catal. 44, 460 (1976). 